\title{
Zur Streitfrage
}

\section{über̉ die Existenz eines Pemphigus acutus.}

Von Dr. Kobner in Breslau.

Nachdem das von Sauvages aufgestellte, in Deutschland hauptsächlich von Wichmann, in Frankreich von Gilibert näher beschriebene Blasenfieber, Febris bullosa s. pemphigodes, Pemphigus acutus, zuerst von Willan und Bateman in Abrede gestellt worden war und nachdem sich gegenüber den Beschreibungen desselben durch neuere Autoren, wie Rayer, Cazenave, Devergie, Gibert, der Widerspruch Hebra's erhoben, welcher, wie die genannten Englinder, verbreitete und auf einander folgende Blasenbildungen auf der Haut nur als chronischen Process ( $P_{0} m p h o l y x$ Willan) gesehen, neinzelne ephemerc Blasen aber, welche er bei fieberhaft Erkrankten, namentlich Pyämischen beobachtet;" nur als accidentelle, unwesentliche Erscheinung hingestellt hatte, suchte v. Bärensprung (CharitéAnnalen, 1862, Bd. X.p. 55 sqq.) die Existenz des Pemphigus acutus durch Analyse zahlreicher fremder und eigener Beobachtungen von Neuem darzuthun. Indess er verfiel hierbei selbst in den Fehler, welchen er "an der geistlos anatomischen Anschauungsweise rügte, welche überall die Krankheitsproducte für die Krankheiten nahm und trotz Wichmann's scharfer Trennung der Febris bullosa von dem chronischen Pemphigus zuletzt dahin führte, dass man jede Blase einen Pemphigus nannte," - er warf Beobachtungen eines allerdings acuten Krankheitsprocesses, dessen gruppirte vesiculöse Eruptionen ausnahmsweise einmal bie und da $z u$ einer Blase zusammenfliessen (Herpes iris), indem er sich nur an das Zustandekommen der Blase hielt, mit Fällen von primärem, acutem, febrilem. Pemphigus zusammen und stellte 
den Herpes iris als die häufigste Form des letzteren, als einen abortiven Pemphigus acutus auf. War es nun schon bezüglich der Charakteristik der Entstehung ler einzelnen Efflorescenzen beim Pemphigus acutus unrichtig, zu behaupten, dass „derselbe nie von vornherein mit grossen Wasserblasen auftrete, sondern dass diese erst durch ein allmäliges Umwachsen und Zusammenfliessen kleinerer, gewöhnlich ringförmig gestellter Bläschen entstehen," so dass B. diese Art der Entwickelung sogar zum diagnostischen Merkmal der Pemphigusblasen erhob: "so hat er überdies das ganz verschiedene Gesammtbild d. h. das ganz verschiedene Verhalten des Gesammtorganismus bei der von früheren Autoren aufgeführten Febris bullosa und bei der von ihm als vesiculöse, theils als bullöse Form derselben angesprochenen Krankheit übersehen und durch diese Unterschätzung der Symptome von Seiten des Gesammtorganismus, auf welche von Wichmann und von den neueren französischen Dermatologen der Hauptaccent bei der gesonderten Aufstellung eines Pemphigus acutus gelegt worden war, die Annahme eines solchen eher discreditirt, als gefördert.

An der Hand dar Erfahrung nun, dass ,beim Herpes iris der übrige Organismus nur sehr wenig betheiligt ist, so dass viele Individuen nur durch die unangenehme Empfindung des Brennens oder Juckens auf denselben aufmerksam werden, dass weder Fieber noch gastrische oder cephalische Symptome bei ihnen auftreten (Hebra)," eine Erfahrung, welche ich bei Individuen, die sei es einmal, sei es bis fünfmal innerhalb 2 bis 3 Jahren an Herpes iris erkrankt waren, stets bestätigt gefunden, trat ich 1864 (in meinen, ,klinischen und experimentellen Mittheilungen a. d. Dermatologie und Syphilidologie," p. 5 u. 6) der Bärensprung'schen Identificirung desselben mit Pemphigus acutus entgegen und bekannte mich aus den dort angegebenen Gründen zu der Ansicht Hebra's, wonach Herpes iris nur eine andere Form (höher gediehene Exsudation) des Erythema papulatum und tuberculatum Willan's (Eryth. exsudativ. multiforme Hebra) ist. Ueber die Existenz eines wirklichen acuten Pemphigus hingegen enthielt ich mich, da mir eigene Erfahrungen nicht zu Gebote standen, des Urtheils. In den letzten Jahren haben mehrere Beobachter, wie Mosler, Thomas (Arch. d. 
Heilkunde, 1868, Hft. 4 ) je einen Fall, Plask u da (Berl. klin. Wochenschr. 1865, Nr. 1 u. 2) und Steffen (ibid. 1866, Nr. 29 bis 32) reihenweise Erkrankungen von Kindern an Pemphigus acutus publicirt and durch einige dieser Beobachtungen ist unserer Ansicht nach die Existenz eines solchen, bei Kindern wenigstens, ausser Zweifel gestellt. Für sein Vorkommen bei Erwachsenen beweisen dieselben freilich nichts und insbesondere kann aus ihnen, welche zumeist Kinder von 1-3 Jahren betreffen, nicht etwa die Symptomatologie eines acuten Pemphigus überhaupt abgeleitet werden. Ueberdies wollen wir auf die Einwände, welche man nach Hebra (Handb. III, 2, p. 269) gegen die Diagnose "acutus" in mehreren dieser Fälle, z. B. in dem von Thomas, erheben kann, nicht näher eingehen.

Wollen wir von acutem Pemphigus bei Erwachsenen sprechen, so müssen wir folgende Postulate crfüllen: Nach kurzen Prodromen und eingeleitet von deutlich ausgesprochenent Fieber, u. z. kurz nach Invasion desselben, nicht in der 2 . oder 3 Woche eines solchen, wie bei Typhus, P'neumonie, mïssen sich direct auf vorher intacter Hant, nicht secundür aus vorherigen Primãrefflorescenzen, wie aus ciner Urticaria, Herpes iris oder Zoster, Blasen entwickeln, welche sich unter Fortdauer oder abwechselnder Exacerbation und Remission des Fiebcrs in wicderholten Nachschïben an verschiedencn Körperstellen, oltne besondere Localisation, erneuern und nach Ablauf von 2 bis 3, höchstens 4 Wochen müssen Fieber und Blasenausbrïche vollständig abgeschbossen sein, ohne dass die lelzteren später, insbesondere in einer fieberfreien Zeit, rïckfällig werden. Wenn wir hiernach diese Art des Auftretens und des Verlaufes, d. h. den Nachweis der engen Verknüpfung und des parallelen Ganges von Blasenausbrüchen und Fieber als nothwendig zur Annahme einer acuten bullösen Phlylitänose zugeben, so glauben wir uns andererseits im einzelnen Falle weder durch das Zusammentreffen des einleitenden Fiebers mit anderen, inneren Veränderungen des Organismus oder überhaupt durch die Art der fiebererregenden Ursache, welche verschieden sein kann, noch durch das gleichzeitige Erscheinen von Dermatitis geringeren Grades (Erythem, Herpesbläschen) an anderen Stellen der allgemeinen Decke in der Diagnose jener beirren lassen zu dürfen. - $\mathrm{Zu}$ dieser Diagnose bestimmte uns zum ersten Mal die folArchiv fiir Dematologie und Syphilis. 1869. 2. 
gende Beobachtung, welche wir im December 1866 an einer Kranken der Breslauer geburtshilfichen Poliklinik mit einem der derzeitigen Praktikanten derselben, Herrn Dr. Boremski, machten und deren Publication uns der Director jener Anstalt, Herr Med. R. Prof. Dr. Spiegelberg in dankenswerther Weise gestattet hat.

Frau Johanna F., Kutschersfrau, 25 Jahre alt, war in. ihrer Jugend, mit Ausnahme von Masern und Seharlach vor dem 7. Jahre, stets gesund gewesen. Die Menstruation trat erst mit dem 20. Jahre auf, kehrte aber seitdem regelmässig, wenn auch spärlich, wieder und danerte 3 bis 4 Tage. Im 22. Jahre verheirathet, gebar sie ein Jahr später einen Knaben, welchen sie selbst säugte und der bis heute gesund blieb. Am 2. oder 3. Tage nach der völlig leichten Entbindung trat nach einem unbedeutenden Fieberanfall ohne äussere Veranlassung, gleichzeitig mit sehr reichlichem allgemeinen Schweisse, Jucken und Röthe am ganzen Körper auf und Tags daraufs im Gesicht, auf der Brust nnd den Extremitäten viele rothe, nur wenig erhabene Flecke, die beim Kratzen auch kleine Bläschen getragen haben sollen und in 8 bis 10 Tagen allmälig erblassten. Seitdem blieb die Haut der Patientin, auch während des ganzen Verlaufes der zweiten, am Abende des 24. November 1866 durch die rechtzeitige und leichte Geburt eines gesunden Mädchens beendigten Schwangerschaft normal. Wenige Tage vor der Niederkunft jedoch empfand Frau F. heftiges Jucken am ganzen Körper. In der Nacht vom 24. zum 25. November bekam sie einen heftigen, lange dauernden Schüttelfrost, der sich innerhalb einiger Standen wiederholte, und dann einer brennenden Hitze wich. Der Lochialfluss stockte. Im Laufe des nächsten Tages steigerte sich die Ernpfindung grosser Hitze in der Haut, besonders als reichlicher Schweiss ausbrach, zn unerträglichem Brennen und Jucken am ganzen Körper, am meisten auf der Fusssohle; die Haut röthete sich daselbst und schon am 26. erhober sich dort und auf einzelnen Punkten der Unterschenkel zuerst erbsen- später bohnengrosse, mit klarer Flüssigkeit prall gefüllte Blasen, denen am 27. November unter ähnlichen Erscheinungen viele andere auf den Armen, der oberen Brustpartie und am 28. auch an den Ober- und Unterschenkeln folgten. In diesen und den folgenden Tagen zeigten sich auch, besonders am Rücken und Nacken, den Vorderarmen und Oberschenkeln viele der unten zu beschreibenden 
rothen erhabenen Flecken. Die Blasen platzten bei dem geringsten äusseren Insult; manche füllten sich wieder, während andere eine rothe, mit einer Epidermisschuppe bedeciste Erosion hinterliessen, welche allmälig eintrocknete. Täglich kamen neue und bedeutendere Blasen. Gastrische Beschwerden waren nicht vorhanden, der Appetit vielmehr gut, das Harnlassen normal, Stuhl nur jeden 2. Tag; dabei stillte Patientin ihr Kind. Erst am 9. Tage der Krankheit, am 3. December 1866, suchte F., weil der Ausschlag unerträglich juckte und den Schlaf raubte, die Hilfe der geburtshilfichen Poliklinik nach. Herr Dr. Boremski notirte ansser der vorstehenden Anamnese folgenden Status praesens: Puls voll, beschleunigt, 112-116, Hauttemperatur etwas erhöht, Zunge wenig belegt, Appetit gut. Das Gesicht, Brust, Rüclien, Extrcmitäten und Plantae ped. mit vielen rothen, im Centrum etwas blasseren, am Saum die Spuren zusammengesunkener kleiner Bläschen tragenden Flecken versehen, auch vielfach zerkratzt. Die Extremitäten sind stellenweise stark, nach Angabe der Kranken erst seit gestern, geschwollen, geröthet und mit zahlreichen Blasen von 1 Linie bis 1 Zoll im Durchmesser und bis $1 / 3$ Zoll Höhe bodeclit. Dieselben entstehen stets sehr schnell, oft binnen einer Stunde, unter dem Gefühl von heftigem Jucken, und enthalten klares, sie prall spanne:ades Serum, während dasselbe in den älteren Blasen schon molkig getrübt ist; sie platzen sehr leicht, oft schon durch die bei Bewegungen der Extremitäten bewirkte Spannung der Haut. Ausser den vorher erwïlnten, auf diese Weise ubrig bleibenden rothen Flecken, deren vertrocknete Epidermis schon losgestossen und deren Cutis nur wenig infiltrirt erscheint, sieht man viele runde oder ovale, am Rande etwas erhabene, durch starke Röthe von der normalen Nachbarschaft sich abhebende Hautpartien, welche theils kreisförmig oder durch Verschmelzung halbkreis- und rosettenförmig sind. Am meisten geröthet und geschwollen ist die Innenseite der Unterschenkel, besonders die Gegend der Knöchel; die Schwellung hat nicht die teigige Beschaffenheit des Oedems, sondern fühlt sich fester und elastischer an. Am 4. December war das Krankheitsbild wesentlich dasselbe, nur waren an einigen Stellen die Blasen geborsten und zusammengefalleu, an anderen neue Nachschübe erfolgt. Diese waren zum Theil erbsenbis bohnengrosse Blasen, zum Theil auch kleinere Bläschen, die auf geröthetem Boden bald in unregelmässigen Gruppen, bald in 


\section{4}

Ringform bei einander standen, in letzterer vor Allem auf dem rechten Arm. Gegen Abend erfolgte, wie auch an mehreren Abenden vorher, ein geringer Schlittelfrost, darauf Hitze und Schweiss, während dessen sich das Brennen beträchtlich steigerte und welchem ein neuer Ausbruch von Blasen und Bläschen folgte; jedoch waren dieselben etwas kleiner als die früheren und weniger zahlreich.

Am 5. December, dem 11. Tage der Erkrankung, als ich in Begleitung des Hru. Boremski die Patientin zuerst sah, befand sie sich bei 108 Pulsen, nur wenig erhöhter Hauttemperatur, etwas geringerer Schwellung der Extremitäten und etwas geringerem Brennen leidlich wohl. Im Ganzen sehr wohl genährt, sah sie etwas bleich und pastös aus, klagte aber nur über das lästige Jucken in der Haut der befallenen Stellen. Im Laufe der vorigen Nacht und des heutigen Morgens waren neue Blasen an der inneren Seite der Handgelenke, der Füsse und dem vorderen Theil der Fusssohlen aufgeschossen - an welchen letzteren sie, wegen der durch die Dicke der Epidermis daselbst bewirkten Spannung, lebhaft schmerzten neue Bläschen besonders auf den Vorderarmen. Die gesammte Oberfläche des Körpers bot sonach folgendes bunte Bild dar.

An den Armen und Beinen besonders stark, demnächst an Brust und Oberbauchgegend, weniger am Halse und nur an wenigen Theilen des unbehaarten Kopfes, nämlich den Ohren and seitlichen Partien der Wangen existirten breite rothe Flecke, theils rundlich, theils von unregelmässigen Conturen neben einer grossen Zahl gruppirter, sowie einzelner Bläschen und nur noch vereinzelten Blasen. Die gesättigt rothen, zum Theil leicht erhabenen Flecke, welche an den Seitentheilen der vorderen Bauchfläche nur schmal - die ganze Nabelgegend blieb bis heute verschont - in der Lenden- und Gesässgegend und an beiden Oberschenkeln hingegen sehr breit waren und in einander flossen, hatten entweder als solche einige Tage verharrt, um dann einzusinken, aber noch als hyperämische Flecke zu persistiren, oder auf ihnen erhoben sich hie und da Knötchen und Bläschen, welche sich zuweilen zu mehreren zusammenfanden, zuweilen aber vereinzelt blieben und zerkratzt wurden - denn sie juckten stark, besonders am Gesäss - oder eintrockneten. Zwischen solchen rothen Flecken, und kleineren, welche zum Theil noch von Fragmenten der Epidermis der geplatzten Blasen bedeckt waren, erhoben sich aber auch auf normaler Haut Bläschen yon Linsengrösse, die, wenn 
vereinzelt, sich sehr bald zu erbsen- bis haselnussgrossen Blasen ausdehnten, wenn in unregelmässigen, lineären oder halbkreis- selbst kreisförmigen, hie und da sogar concentrischen Gruppen auf rother, leicht erhabener Basis aufschiessend, nie zu Blasen wurden, sondern eintrockneten. An den Oberarmen, Unterschenkeln und zwischen den Zehen überwogen einzelne Blasen an Zahl die Bläschen und Bläschengruppen (Herpes phlyctaenodes); an den Vorderarmen, Oberschenkeln, dern Hals und der Seitenwangengegend hingegen waren letztere viel zahlreicher. Der Rücken, dessen obere Hälfte viel wenigẹ als, wie erwähnt, die untere befallen war, zeigte viele Spuren der kratzenden Nägel, aber keine Bläschen oder Blasen; in der Gegend des rechten Schulterblattes stellte sich eine etwa handtellergrosse, dunkelrothe; infiltrirte, offenbar aus mchreren lieineren entstandene Rosettenfigur mit schon etwas runzliger Epidermis dar, welche einem Erythema papulatum, respective gyratum glich, während die uber handbreiten rothen Flächen, welche sich continuirlich von der Lenden- bis in die seitliche Unterbauchgegend jeder Scite erstreckten und nur die Haut zu beiden Seiten der Wirbelsäule intact liessen, minder scharfe Conturen besassen. Die Schleimhaut der Mund- and Rachenhöhle, sowie der Augenlieder war ganz normal. Die Lochien hatten sich, trotzdem seit zwei Tagen (bei Verabreichung von Morphium allabendlich wegen des Juckens bei Nacht, Citronenlimonade am Tage) das Fieber sehr nachgelassen, noch nicht eingefunden. Die Milch floss reichlich aus den gut gefüllten Brüsten. Urin ziemlich reichlich, klar, ohne Sedimente und frei von Eiweiss. - Am Abende erfolgte Exacerbation des Fiebers mit Frost, Hitze und Schweiss and nach letzterem erschienen auf frisch gerötheten Stellen, besonders an den Hand- und Fussgelenken einige erbsen- bis bohnengrosse Blasen. -

Am Morgen des 6. December war der Puls auf 92 gesunken, die Hauttemperatur wenig erhöht, die Schwellung der oberen Extremitäten hatte etwas abgenommen, die Blasen an den Oberarmen waren fast sämmtlich geborsten und die geröthete Bạsis derselben secernirte nur noch wenig, an den Vorderarmen waren die meisten derselben schon trocken und ihre Epidermis abgefallen, auch viele Bläschengruppen eingesunken und ihr Boden. blässer, gelbröthlich geworden. Dagegen waren andere mit Bildung ähnlicher Figuren aufgeschossen, welche jedoch, gleich den frischen Blasen am Handgelenk, kleiner als die früheren blieben. Die Unterschenkel blässer 
als am vorigen Abend, aber besonders auf dem Fussrücken, etwas ödematös; auf ihrer vorderen Fläche erübrigten nur noch Spuren von Blasen und Bläschen, rlagegen mehr an der hinteren und inneren Fläche. Die Blasen auf der Fusssohle und zwischen den Zehen waren flach und faltig geworden. Am Abend keine Exacerbation, keine neuen Blasen, vielmehr einige auf den Forderarmen und Handgelenken collabirt.

Vom 7. December, also vom 13. Krankheitstage an, an welchem das Fieber gänzlich aufgehört (Morgens 80, Abends 88 P., normale Temperatur) und auch Abends sich nur stärkeres Brennen, besonders in den unteren Extremitäten, aber ohne folgende Efflorescenzen einstellte, heilten binnen etwa 6 Tagen alle die beschriebenen Hauteruptionen $a b$ und verriethen sich am 13. December nur noch durch zerstreute rothe Fleckenreste; auch war die Haut der Extremitäten wieder völlig abgeschwollen. Am 13. Dec. zeigten sich einzelne Furunkel an der Oberlippe und im Naken, denen am 14. und 15. mebrere andere an den Extremitäten folgten; jedoch blieb ihre Zahl und Grösse so gering, dass sie gegen den 22. Dec. schon geschwunden waren. Eine gleichzeitig eintretende Diarrhöe wurde durch Opium schnell beseitigt. - Zur Nachcur hatte Patientin, seitdem sie fieberfrei war, Eisenpräparate erhalten. -

Ftwa nach 4 Monaten wiedergesehen, gab Frau F. an, ab und $z u$ noch einen Blutschwär bekommen zu haben; sonst war sie aber munter, sah, so weit man dies in der Souterrainwohnung sein konnte, welche sie seit Jahresfrist inne hatte, frisch aus und säugte ibr gut gedeihendes Kind. Seit der Aufnahme dieser Beobachtung haben wir mehr als 2 Jahre verstreichen lassen, um einestheils zu constatiren, ob. die Blasenausbrüche spnntan wiederkehren würden - was bis jetzt nicht der Fall war - und ferner, wie es sich damit bei Beendigung einer etwaigen neuen Schwangerschaft verhalten würde. Diese "gute Hoffnung" ist jedoch bisher der Patientin und zu unserem Bedauern auch uns nicht erfüllt worden.

Es ist klar, dass die an der Haut des ganzen Körpers so ncut aufgetretenen und abgelaufenen Eruptionen in diesen Falle ebenso wie das starke Fieber, welches ihrem ersten Auftreten, sowie jedem folgenden erheblicheren Nachschube unmittelbar vorauging, mit der Eutbindung der. Frau in nächstem Zusammenhang standen. Schon das Auftreten einer wie Urticaria beschrie- 
benen, freilich von nur unbedeutendem Fieberanfall eingeleiteten und nur eine Woche bestehenden Eruption im ersten Wochenbett müsste darauf führen. Nun kann man sich, auf Grund der Wahrnehmung, dass fast stets ein heftiger Fieberfrost voranging und die Hautveränderungen erst im Stadium des starken Schweisses sich zeigten, vorausgesetzt, dass man inber den frisch entleerten Uterus als Ausgangspunkt des Fiebers einig ist, das schnelle Zustandekommen der Bläschen, Blisen und der übrigen Exsudationsflecken der Haut auf doppelte Art denken: entweder als metastatische Depôts, d. h. durch Verstopfung kleiner arterieller und capillärer Hautgefässe durch Einboli, deren Quelle man in den Uterinvenen zu suchen hätte, welcher durch den Schüttelfrost sich kundgebenden Embolie sofort collaterale Hyperämie und Transsudation folgte, ein Hergang, wie ihn Bohn jüngst beim Erythema nodosum und der Peliosis rbeumat. Wahrscheinlich zu machen gesucht hat, ohne jedoch noch das erste Desiderat, den Nachweis der embolischen Bildungsstätte, erfüllen zu könnenn oder als cutane Angioneurose (Eulenburg), wobei von den Uterinnerven aus die vasomotorischen Nerven der Haut reflectorisch gereizt werden und zunächst starke Contraction der Hautgefässe (Cutis anserina und Schüttelfrost), alsdann Relaxation derselben mit sofortiger Hyperämie und bald darauf folgender, verschiedene Grade erreichender Exsudation bewirken können. Wir wollen auf die Entscheidung dieser Alternative nicht weitläufig eingehen, sondern nur einige Wahrnehmungen an der Kranken nachtragen, welche uns gegen die erstere Annahme bestimmen, also gegen eine Febris puerperalis mit Metastasen auf die Haut und für eine Febris in puerperio sprechen, welches bei der reizbaren Haut der Kranken zu einer „F. vesicularis et bullosa" wurde. Weder während, noch nach der Niederkunft hatte Pat, auch nur die geringsten Beschwerden von Seiten des Uterus; derselbe und seine Nachbarschaft zeigte nicht die geringste Druckempfindlichkeit, keine übelriechende Absonderung uud involvirte sich rechtzeitig. Kein inueres Organ erkrankte, wie es doch bei der Annahme von Hunderten im Blute kreisenden Gerinnseln zu erwarten gewesen wäre. Der relativ gute Appetit in der apyretischen Zeit, die wenig belegte Zunge, das immer klare Sensorium, die fortbestehende Milchsecretion passen nicht 
in das Bild eines Puerperalfiebers. Dahingegen hat die zweite Ansicht die Antecedentien der Kranken für sich, sowie sich aus dieser Reizbarkeit der Haut die noch wochenlang nach Ablauf der acuten Phlyktaenen hie und da sich bildenden Furunkel erklären. Verhält sich aber die Sache so, so kann man fragen, ob dieser Fall wohl die Bezeichnung Pemphigus acutus rechtfertigt. Wir bejahen dies 1 . per analogiam mit den fieberhaften Formen des Herpes und der Urticaria, sowie ferner mit einer Art des chronischen Pemphigus, den man, weil er bei manchen Frauen constant nach jeder Conerption auftritt und mit dem Ende der Schwangerschaft aufhört (Hardy, Hebra), ausdrücklich Pemphigus hystericus genannt hat. 2. So gut wie in vorstehender Beobachtung von den Uterinnerven aus, so kann in anderen Fällen durch anderweitige, nur nicht immer in ihrer Quelle zu eruirende Reizung der Gefissnerven der Haut acuter Pemphigus erzengt werden. So verschieden und meistens dunkel der causale Nexus, so constant wird in den spärlichen zuverlässigen Beobachtungen eines solchen, uuter welchen wir z. B. diejenige von $\mathrm{Hebra}$ selbst an einem 13jährigen Tischlerlehrling gemachten (Zeitschr. der Wiener Aerzte, Jahrg. X) erwähnen, immer eine besondere Disposition der erkrankten Individuen zu Hautkrankkeiten angeführt. Wenn nun trotz des gewiss häufigen Auftretens von Fiebern bei Individuen mit solcher Disposition der Haut der Pemphigus acutus universalis eine grosse Seltenheit ist, so dürfen wir nichts anderes daraus schliessen, als dass es noch nothwendige Bedingungen für die Genese desselben, vermuthlich in der Haut selbst gibt, welche uns entgehen. Aus der Verschiedentrtigkeit der Ursachen und aus Variationen im Verlauf aber (cf. Bazin, Leçons s. les affect. cutan. de nature arthrit. et dartreuse, Paris, 1860, p. 128 und 282) glauben wir ebenso wenig seine gesonderte Aufstellung verwerfen zu dürfen, wie die mancher anderer Formen von Hautkraukheiten, acuter (der oben genannten Angioneurosen) wie chronischer (vieler Fälle von Pompholix), deren mehr als symptomatische Bedeutung durchaus uicht feststeht und bei welchen letzteren namentlich wir die Krankheit ausschliesslich in der hervorragend betheiligten Haut annehmen und sie nack der Form der Eruption benennen, weil wir noch nicht im Stande sind, die fernerliegenden ursächlichen Beziehungen nachzuw eisen. 